\title{
The Protein Data Bank and Its Uses in Structural Biology Education
}

\author{
underlineJudith Voet $^{1}$, Shuchismita Dutta ${ }^{2}$
}

${ }^{1}$ Department of Chemistry and Biochemistry, Swarthmore College, 500 College Avenue, Swarthmore, PA 1908. ${ }^{2}$ Research Collaboratory for structural Bioinformatics, Department of Chemistry and Chemical Biology, Rutgers, The State University of New Jersey, 610 Taylor Road, Piscataway, NJ 08854-8087

The Protein Data Bank (PDB) is a repository for the structures of proteins and nucleic acids. It contains files of their 3-dimensional coordinates, information on how these structures were determined and references to the journal articles describing them. The PDB was established in 1971 by Helen Berman (it 's present director) and has grown exponentially so that it now contains $\sim 25,000$ data files representing X-ray crystallographic, NMR and other structure determinations. Database querying and data miningtools and resources at the PDB make it possible to search, compare and infer or predict the function of newly identified proteins. Computer graphics capabilities make it possible for anyone to easily visualize and study the structural data. The capability to present beautiful graphic representations of the 3-dimesnional structures of proteins and nucleic acids has been a boon to the education community. Communicating an understanding of these structures and the chemical forces determining them and their interactions is one of the major aims of biochemistry and molecular biology education. The ability to teach these principles visually has made a great difference in our ability to excite our students and provide them with physical interpretations for some abstract concepts in biochemistry and molecular biology. In this talk we will explore some of the ways that the education community uses the PDB. 